\title{
A retrospective study regarding the method of delivery of adolescents in a Romanian Hospital
}

\author{
ANA VERONICA UZUNOV ${ }^{1,2^{*}}$, ROXANA ELENA BOHILTEA ${ }^{2}$, OCTAVIAN MUNTEANU $^{3 *}$, \\ DRAGOS NEMESCU ${ }^{4}$ and MONICA MIHAELA CIRSTOIU ${ }^{2}$
}

\author{
${ }^{1}$ Department of Obstetrics and Gynecology, 'Carol Davila' University of Medicine and Pharmacy \\ Doctoral School, University Emergency Hospital Bucharest; ${ }^{2}$ Department of Obstetrics and Gynecology, \\ 'Carol Davila' University of Medicine and Pharmacy, University Emergency Hospital Bucharest, \\ 050098 Bucharest; ${ }^{3}$ Department of Anatomy, 'Carol Davila' University of Medicine and Pharmacy, 050474 Bucharest; \\ ${ }^{4}$ Department of Obstetrics and Gynecology, 'Grigore T. Popa' University of Medicine and Pharmacy, 700115 Iasi, Romania
}

Received April 17, 2020; Accepted May 19, 2020

DOI: $10.3892 /$ etm.2020.8835

\begin{abstract}
Adolescent pregnancies are associated with a high number of risks for the newborn and mother. Hence, an increased number of emergency caesarean extractions are performed in this group of patients. The aim of this study was to analyze the pregnancy-related conditions, the way of delivery and the neonatal outcome of all the patients who delivered in the Department of Obstetrics and Gynecology of University Emergency Hospital in Bucharest, a tertiary unit in Romania in a period of 5 years. An observational, retrospective study was performed on a group of 686 patients, aged 12 to 19 years, who delivered in the Department of Obstetrics and Gynecology of University Emergency Hospital in Bucharest, between January 1, 2014 and December 31, 2018. The pregnant women were divided into two age groups. In the first group were pregnant patients aged under 18 years, and in the second group pregnant adolescents between 18 and 19 years. Whether the patients underwent prenatal screening was analyzed. Furthermore, the age of the patients, the rate of caesarean extraction and vaginal birth and the obstetrical complications were evaluated and compared. The outcome of the newborns was assessed based on Apgar score at $1 \mathrm{~min}$ and birth weight. Regarding the results, 464 of the 686 patients did not undergo any medical visits during pregnancy. In total, $52.76 \%$ of the patients delivered by caesarean section. The most frequent indications for caesarean extraction, in both analyzed groups, were Cephalo-pelvic disproportion, fetal distress and uterine
\end{abstract}

Correspondence to: Dr Roxana Elena Bohiltea, Department of Obstetrics and Gynecology, 'Carol Davila' University of Medicine and Pharmacy, University Emergency Hospital Bucharest, 169 Splaiul Independenței, 050098 Bucharest, Romania

E-mail: r.bohiltea@yahoo.com

*Contributed equally

Key words: adolescents, pregnancy, caesarean section, fetal outcome, cephalo-pelvic disproportion, fetal distress, uterine scar scar after caesarean section. The lack of specific protocols regarding the ante-, peri- and post-natal management of adolescents is probably the cause of the alarmingly increasing number of patients pertaining to the group who deliver by caesarean section.

\section{Introduction}

Teenage pregnancy is defined by the United Nations Children's Fund (UNICEF) as being pregnant between the age of 13 and 19 years (1). Each year, all over the world 16 million teenage women give birth each year; this represents $\sim 11 \%$ of all births (1). The real problem is that $95 \%$ of all these births occur in low, and middle income countries (1). Romania occupies the second place in Europe in teenage pregnancy (1).

Adolescent pregnancies are associated with a high number of risks for the newborn and mother $(2,3)$. The pathologies associated with teenage pregnancy are: Preterm delivery, low birthweight, gestational hypertension, preeclampsia-eclampsia and neonatal death (4-7). Due to these conditions, an increased number of emergency caesarean extractions is performed in adolescent pregnant patients $(2,4,5)$.

It is estimated that the mortality due to obstetrical complications in a pregnant adolescent is twice that in a woman between 20 and 30 years (8). Obstetrical related pathologies are the main cause of mortality in teenagers (8-11). It is estimated that $27.5 \%$ of the maternal deaths in this group of patients occur after vaginal delivery, $18.7 \%$ due to complications of pregnancy, $9.4 \%$ due to other maternal care related to the fetus and $5.4 \%$ of deaths due to abortion (8).

Gestational diabetes and fetal anomalies are also relatively frequent in teenage pregnancy (2). In a retrospective cohort study 2 groups of pregnant patients were analyzed and compared: A group of adolescents aged 11-19 years and a second group of patients aged 20-24 years were included (2). In this study the incidence of gestational diabetes, fetal anomalies and preeclampsia were compared. Torvie et al (2) demonstrated that preeclampsia, gestational diabetes and fetal anomalies are more frequent in young teenagers aged 11-14 years). However, caesarean extraction was more rare 
in adolescents under 17 years at $28.1 \%$, increasing with age, reaching $36.2 \%$ between $18-24$ years (2).

Pregnancy during adolescence represents an important challenge also for the physical status of the patient $(4,12)$. As adolescence is a transitional period of physical and psychological development between childhood and adulthood, pregnancy at this age can determine imbalances that can be transitory or can become chronic if they are not properly managed (12).

The aim of this study was to analyze the pregnancy-related conditions, the way of delivery and the neonatal outcome of all the patients who delivered in the Department of Obstetrics and Gynecology of University Emergency Hospital in Bucharest, a tertiary unit in Romania in a period of 5 years.

\section{Patients and methods}

An observational, retrospective study was performed on a group of 686 patients, aged 12-19 years, who delivered in the Department of Obstetrics and Gynecology of University Emergency Hospital in Bucharest, between January 1, 2014 and December 31, 2018. The pregnant women were divided into two age groups. In the first group pregnant patients aged under 18 years were analyzed, the group represented patients who had not reached legal adulthood while in a second group pregnant adolescents aged 18-19 years. The information regarding pregnancy, delivery outcomes and neonatal data were retrieved from the hospitalization sheets and the Base Data System of University Emergency Hospital in Bucharest.

Maternal age was defined as that recorded at the time of delivery. Expulsions of the gestational product after completed 24 weeks were defined as deliveries, while before 24 weeks were considered abortions and were not included in this study.

It was first analyzed if the patients in the two groups underwent routine medical visits during pregnancy (defined as prenatal screening) which include: physical examination, fetal ultrasound scan and laboratory tests.

The age of the patients were compared and the rate of caesarean extraction and vaginal birth, the obstetrical complications and the management of patients were analyzed in the two groups. The outcome of the newborns was assessed based on Apgar score at $1 \mathrm{~min}$ and birth weight.

\section{Results}

A total of 686 adolescents were included in study. Three hundred and forty-three patients aged 12-17 years were analyzed in group A, while the others aged 18-19 years formed group B.

First it was evaluated if the patients included into the two age groups underwent routine medical visits (Fig. 1). An enormous percent of patients included in this study did not undergo any routine medical visits, $94.46 \%(n=324)$ of the patients in group $A$ and $40.81 \%(n=140)$ in group B did not undergo any prenatal screening (Fig. 1).

Analyzing the delivery method (Fig. 2), an approximately equal number of vaginal spontaneous deliveries were recorded $(n=173)$ and caesarean extraction $(n=170)$ in patients from group A. In group B caesarean section was performed in $55.97 \%(n=192)$ of the cases. The rest of the patients delivered vaginally; instrumental delivery was performed in 5 cases, forceps delivery in 4 cases and one vacuum assisted delivery.

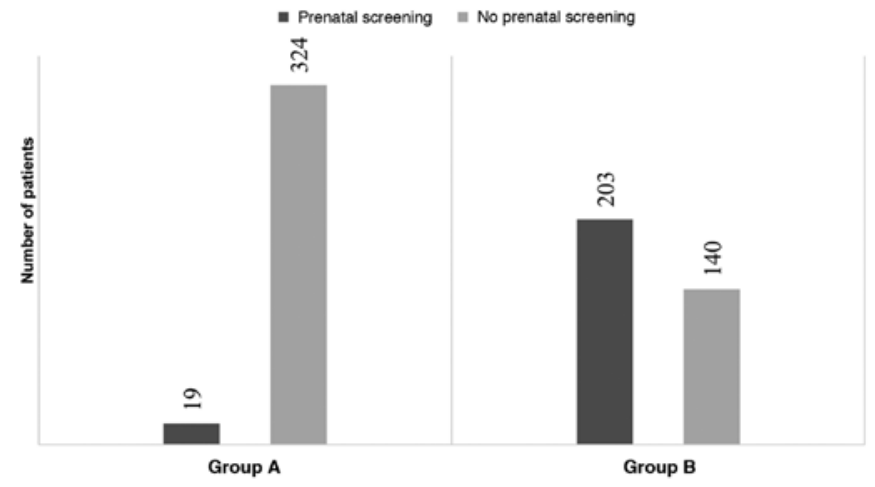

Figure 1. Distribution of patients included in the study according to prenatal screening.

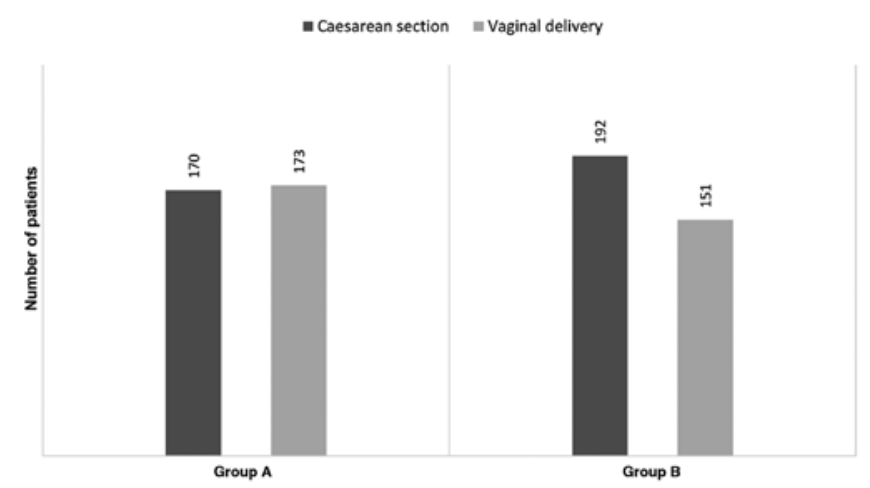

Figure 2. Method of delivery in the two analyzed groups.

The indications of caesarean extraction of patients evaluated in the two groups were analyzed and compared (Table I). The most frequent indication for caesarean extraction was cephalo-pelvic disproportion, while fetal distress was the second most common indication, in both analyzed groups (Table I).

Surprising, the third most frequent indication for this type of delivery in entire lot of adolescents analyzed was the high risk of rupture of uterine scar after caesarean section, 27 patients from each group. The youngest patient with a uterine scar after caesarean section was a 16-year old patient.

It is important to mention that in the category of 'Other' (indication for caesarean section) of both groups included patients with various obstetrical and non-pregnancy-related pathologies. Group A patients diagnosed with umbilical cord prolapse, HIV positive, uterine malformations, lymphomas, or other operated malignancies were included. Group B included patients diagnosed with fetal malformations, retinal dystrophy, or severe depression.

The outcome of the neonates was evaluated using the Apgar score at $1 \mathrm{~min}$. The neonatal status of all the fetuses that were delivered by patients pertaining to both groups were analyzed and compared (Table II).

Table II shows that most patients analyzed delivered a fetus who received a calculated Apgar score of 9; a good immediate evolution. The mean Apgar score was 8.69 and the mean Apgar score for group A was 8.78 and for group B was 8.60. It was noted that twice as many of the patients in group B delivered 
Table I. Distribution of the patients in the two analyzed groups according to the indication for caesarean section.

\begin{tabular}{lcr}
\hline Indication of caesarean section & Group A (n=170), \% (n) & Group B (n=192), \% (n) \\
\hline Cephalo-pelvic disproportion & $25.88(44)$ & $21.3(41)$ \\
Fetal distress & $20.59(35)$ & $18.7(36)$ \\
Uterine Scar after caesarean section & $15.88(27)$ & $14.06(27)$ \\
Dystocic presentation & $7.65(13)$ & $11.46(22)$ \\
Preeclampsia & $6.47(11)$ & $7.81(15)$ \\
Placenta Praevia & $3.53(6)$ & $5.21(10)$ \\
Negative induction of labor & $8.24(14)$ & $10.94(21)$ \\
Intrauterine growth restriction & $2.35(4)$ & $3.65(7)$ \\
Other & $9.41(16)$ & $6.77(13)$ \\
\hline
\end{tabular}

Table II. Distribution of the patients in the two analyzed groups according to the Apgar score of the fetus at 1 min and according to the method of delivery in each group.

\begin{tabular}{|c|c|c|c|c|c|c|}
\hline \multirow[b]{2}{*}{$\begin{array}{l}\text { Apgar } \\
\text { score }\end{array}$} & \multicolumn{3}{|c|}{ Group A ( $n=343$ cases) } & \multicolumn{3}{|c|}{ Group B ( $n=343$ cases) } \\
\hline & $\begin{array}{c}\% \text { of } \\
343 \text { cases (n) }\end{array}$ & $\begin{array}{c}\text { Caesarean } \\
\text { section } \\
\% \text { of } \\
170 \text { cases (n) }\end{array}$ & $\begin{array}{c}\text { Vaginal } \\
\text { delivery } \\
\% \text { of } \\
173 \text { cases }(n)\end{array}$ & $\begin{array}{c}\% \text { of } \\
343 \text { cases (n) }\end{array}$ & $\begin{array}{c}\text { Caesarean } \\
\text { section } \\
\% \text { of } \\
192 \text { cases (n) }\end{array}$ & $\begin{array}{c}\text { Vaginal } \\
\text { delivery } \\
\% \text { of } \\
151 \text { cases (n) }\end{array}$ \\
\hline 1 & $0.87(3)$ & $1.17(2)$ & $0.57(1)$ & $1.16(4)$ & $1.56(3)$ & $0.66(1)$ \\
\hline 2 & 0 & 0 & 0 & $0.29(1)$ & 0 & $0.66(1)$ \\
\hline 3 & 0 & 0 & 0 & 0 & 0 & 0 \\
\hline 4 & 0 & 0 & 0 & $0.58(2)$ & 0 & $1.32(2)$ \\
\hline 5 & $0.58(2)$ & $1.17(2)$ & 0 & $0.87(3)$ & $0.52(1)$ & $1.32(2)$ \\
\hline 6 & $1.74(6)$ & $2.35(4)$ & $1.15(2)$ & $2.33(8)$ & $4.17(8)$ & 0 \\
\hline 7 & $3.79(13)$ & $4.11(7)$ & $3.46(6)$ & $6.7(23)$ & $6.77(13)$ & $6.62(10)$ \\
\hline 8 & $20.11(69)$ & $22.35(38)$ & $17.91(31)$ & $17.2(59)$ & $17.19(33)$ & $17.22(26)$ \\
\hline 9 & $51.89(178)$ & $57.64(98)$ & $46.24(80)$ & $54.81(188)$ & $63.54(122)$ & $43.71(66)$ \\
\hline 10 & $20.99(72)$ & 11.17 (19) & $30.63(53)$ & $16.03(55)$ & $6.25(12)$ & $28.48(43)$ \\
\hline
\end{tabular}

a baby with an Apgar score between 1 and 5, compared with group A.

Giving the fact that fetal distress was the second most common indication for caesarean section in both analyzed groups we were interested to evaluate if there are significant differences of fetal outcome (interpreted in relation with Apgar score) and the method of delivery (Table II). As shown in Table II no significant differences of Apgar score were noted in relation with the method of delivery.

The weight of the fetuses of the patients were also analyzed and compared in the two groups (Table III). The newborns of patients pertaining to both groups were distributed into 8 categories of fetal weight.

Table III shows that there are no significant differences between the weights of fetuses of patients evaluated in the two groups, according to the 8 pre-formed categories.

Giving the fact that cephalo-pelvic disproportion was the most frequent indication for caesarean extraction in both analyzed groups we were interested to evaluate if there are significant differences between the fetal weight and the method of delivery. The mean weight was $3.028 \mathrm{~g}$, but
$20 \%$ of all patients delivered a fetus with a weight under 2,500 g (Table III). The distribution of patients in group B according to fetal weight is interesting; $19.77 \%$ of the patients who delivered vaginally had a fetus that weighted $>3,500 \mathrm{~g}$, while only $13.02 \%$ of the patients who delivered by caesarean section had a fetus with the same weight. A significant difference was seen in category 2,000-2,500 g, approximately a double number of patients delivered by caesarean section in group B. In group A all fetuses weighing over 4,000 g were delivered by caesarean section.

In Fig. 3 it is shown that most of the analyzed teenagers gave birth to fetuses weighing between 3,000-3,499 g, even the youngest adolescent, who was 12 years old. Furthermore, even mothers with low age delivered newborns weighing $>3,500 \mathrm{~g}$.

\section{Discussion}

Between January 1, 2014 and December 31, 2018, 686 patients, aged between 12 and 19 years, delivered in the Department of Obstetrics and Gynecology of University Emergency Hospital in Bucharest, Romania. Unfortunately, 464 of these patients, 
Table III. Distribution of the patients in the two analyzed groups according to the weight of the fetus and according to the method of delivery in each group.

\begin{tabular}{|c|c|c|c|c|c|c|}
\hline \multirow[b]{2}{*}{$\begin{array}{l}\text { Newborn } \\
\text { weight }(\mathrm{g})\end{array}$} & \multicolumn{3}{|c|}{ Group A ( $\mathrm{n}=343$ cases) } & \multicolumn{3}{|c|}{ Group B ( $\mathrm{n}=343$ cases $)$} \\
\hline & $\begin{array}{c}\% \text { of } \\
343 \text { cases (n) }\end{array}$ & $\begin{array}{c}\text { Caesarean } \\
\text { section } \\
\% \text { of } \\
170 \text { cases }(n)\end{array}$ & $\begin{array}{c}\text { Vaginal } \\
\text { delivery } \\
\% \text { of } \\
173 \text { cases (n) }\end{array}$ & $\begin{array}{c}\% \text { of } \\
343 \text { cases (n) }\end{array}$ & $\begin{array}{c}\text { Caesarean } \\
\text { section } \\
\% \text { of } \\
192 \text { cases (n) }\end{array}$ & $\begin{array}{c}\text { Vaginal } \\
\text { delivery } \\
\% \text { of } \\
151 \text { cases (n) }\end{array}$ \\
\hline$<1,000$ & $0.29(1)$ & 0 & $0.57(1)$ & $0.58(2)$ & $0.52(1)$ & $0.66(1)$ \\
\hline $1,000-1,499$ & $0.87(3)$ & $1.18(2)$ & $0.57(1)$ & $1.45(5)$ & 0 & $3.31(5)$ \\
\hline $1,500-1,999$ & $1.74(6)$ & $2.35(4)$ & $1.15(2)$ & $1.74(6)$ & $2.08(4)$ & $1.32(2)$ \\
\hline $2,000-2,499$ & $11.95(41)$ & $11.18(19)$ & $12.71(22)$ & $5.83(20)$ & $8.85(17)$ & $1.99(3)$ \\
\hline $2,500-2,999$ & $34.69(119)$ & $32.94(56)$ & $36.41(63)$ & $29.44(101)$ & $34.9(67)$ & $22.52(34)$ \\
\hline $3,000-3,499$ & $36.15(124)$ & $38.82(66)$ & $33.52(58)$ & $44.89(154)$ & $40.63(78)$ & $50.33(76)$ \\
\hline $3,500-4,000$ & $13.7(47)$ & $12.35(21)$ & $15.02(26)$ & $14.57(50)$ & $10.94(21)$ & $19.21(29)$ \\
\hline$>4,000$ & $0.58(2)$ & $1.18(2)$ & 0 & $1.45(5)$ & $2.08(4)$ & $0.66(1)$ \\
\hline
\end{tabular}



Figure 3. Number of newborns according to the weight of fetuses and age of the mother.

$94.46 \%$ of the adolescents aged between 12 and 17 years and $40.81 \%$ of the teenagers aged between 18 and 19 years did not undergo any routine medical visits during pregnancy (Fig. 1). This aspect is particularly important because, as already highlighted by other specialists, lack of medical visits during pregnancy in adolescent population involves multiple adverse birth outcomes increasing both maternal and fetal morbidity and mortality $(13,14)$. The socioeconomic status of these patients can also increase the risks highlighted above $(13,14)$.

In our study a high rate of caesarean sections was observed as a method of delivery in adolescent pregnant patients. Half of the teenagers aged between 12 and 17 years delivered by caesarean section. In the group of patients aged between 18 and 19 years we observed an even higher rate of caesarean extraction, $55.97 \%$ of the cases (Fig. 2). Torvie et al (2), in a study in which 8,028 patients were included, also observed that caesarean extraction is less frequent in patients aged between 15 and 17 years. However, in another retrospective study, on a period of 37 years, in which patients under 20 years were included, concluded that women under 20 years had a significantly higher rate of vaginal delivery compared with women aged between 20 and 30 years (15).

In our study, in both analyzed groups, the most frequent indication for caesarean extraction was cephalo-pelvic disproportion (Table I). However, we did not detect any significant differences between the fetal weight and the method of delivery (Table III). Cephalo-pelvic disproportion is one of the most common indications for caesarean extraction (16). However, in adolescent population, rigorous criteria for establishing this diagnosis and therefore caesarean extraction still need to be evaluated. Jain and Gupta also observed, in a study on 100 adolescent patients, that cephalo-pelvic disproportion was the most frequent indication for caesarean section (17).

In our study, in both analyzed groups, the second most frequent indication for caesarean extraction was fetal distress (Table I). Similarly to our conclusion, Jain and Gupta (17) also observed that fetal distress was the second most frequent indication for caesarean section $(25 \%$ of all caesarean sections). The fetal outcome was interpreted in relation with the Apgar score. However, no significant differences of Apgar score were noted in relation with the method of delivery (Table II). Adequate management and prompt decision to extract the fetus by caesarean section decreased the risk of short-, mid- and probably even long-term fetal complications. Other authors also demonstrated that emergency caesarean section in adolescents with fetal distress could decrease fetal morbidity and mortality (18).

An important number of patients in our study, who delivered by caesarean section were included in the category of 'Other' (indication for caesarean section). All these patients had various obstetrical and non-pregnancy-related pathologies. From group A we included in this category patients diagnosed with: umbilical cord prolapse, HIV positive, uterine malformations, lymphomas, or other operated malignancies. From group B we included in this category patients diagnosed with fetal malformations, retinal dystrophy, or severe depression. University Emergency Hospital in Bucharest is a multidisciplinary medical unit and the Department of Obstetrics and Gynecology is a tertiary center that manages a high number of pregnant adolescents with rare conditions or important comorbidities, which require caesarean delivery in many cases.

In the present study, in both analyzed groups, the third most frequent indication for caesarean extraction was uterine scar after caesarean section (Table I). As previously discussed 
an important number of adolescents deliver by caesarean section in first pregnancy. The risk of uterine rupture among patients who try to deliver vaginally with a uterine scar after caesarean section has already been demonstrated in adult population (19). However, there are no significant statistical studies regarding this matter in adolescent population. This is why most obstetricians recommend delivery by caesarean section if an adolescent has a uterine scar.

Regarding the results, 464 of the 686 patients analyzed in our study on adolescent pregnancy did not undergo any medical visits during pregnancy (Fig. 1). Moreover, most of these patients presented at the Emergency Room in advanced stages of labor. As shown caesarean extraction was performed in an important number of cases (Fig. 2). However, the fetal outcome was very good in the majority of cases (Table II). The lack of specific protocols regarding the ante-, peri- and post-natal management of adolescents is probably the cause of the alarmingly increasing number of patients pertaining to this group who deliver by caesarean section. This is why future study is required in this field that is a major health problem worldwide.

In conclusion, of the 686 patients, aged between 12 and 19 years, who delivered in the Department of Obstetrics and Gynecology of University Emergency Hospital in Bucharest, 464 did not undergo any routine medical visits during pregnancy.

Half of the teenagers aged between 12 and 17 years delivered by caesarean section. In the group of patients aged between 18 and 19 years an even higher rate of caesarean extraction at $55.97 \%$ of the cases was observed. The most frequent indications for caesarean extraction were cephalo-pelvic disproportion, fetal distress and uterine scar after caesarean section.

The lack of specific protocols regarding the ante-, peri- and post-natal management of adolescents is probably the cause of the alarmingly increasing number of patients pertaining to the group who deliver by caesarean section.

\section{Acknowledgements}

Not applicable.

\section{Funding}

No funding was received.

\section{Availability of data and materials}

The datasets used and analyzed during the current study are available from the corresponding author on reasonable request.

\section{Authors' contributions}

AVU and OM collected, analyzed and interpreted the patient data regarding adolescent pregnancy and the outcome of delivery. REB, DN and MMC were involved in the conception of the study and substantially contributed to the writing of the manuscript. All authors read and approved the final manuscript.

\section{Ethics approval and consent to participate}

Not applicable.

\section{Patient consent for publication}

Not applicable.

\section{Competing interests}

The authors declare that they have no competing interests.

\section{References}

1. Cook SM and Cameron ST: Social issues of teenage pregnancy. Obstet Gynecol Reprod Med 25: 243-248, 2015.

2. Torvie AJ, Callegari LS, Schiff MA and Debiec KE: Labor and delivery outcomes among young adolescents. Am J Obstet Gynecol 213: 95.e1-95.e8, 2015.

3. Fraser AM, Brockert JE and Ward RH: Association of young maternal age with adverse reproductive outcomes. N Engl J Med 332: 1113-1117, 1995.

4. Gortzak-Uzan L, Hallak M, Press F, Katz M and Shoham-Vardi I: Teenage pregnancy: Risk factors for adverse perinatal outcome. J Matern Fetal Med 10: 393-397, 2001.

5. Chen XK, Wen SW, Fleming N, Demissie K, Rhoads GG and Walker M: Teenage pregnancy and adverse birth outcomes: A large population based retrospective cohort study. Int J Epidemiol 36: 368-373, 2007.

6. Ganchimeg T, Ota E, Morisaki N, Laopaiboon M, Lumbiganon P, Zhang J, Yamdamsuren B, Temmerman M, Say L, Tunçalp Ö, et al; WHO Multicountry Survey on Maternal Newborn Health Research Network: Pregnancy and childbirth outcomes among adolescent mothers: A World Health Organization multicountry study. BJOG 121 (Suppl 1): 40-48, 2014.

7. Hoque $\mathrm{M}$ and Hoque S: Comparison of perinatal and obstetrics outcomes among early adolescents, late adolescents and adult pregnant women from rural South Africa. East Afric J Pub Health 7: 171-176, 2010.

8. González-Andrade F and Saeteros-Cordero X: Pregnancy in adolescence and adverse neonatal outcomes in Ecuadorian mestizo newborns. Pediatr Neonatol 61: 216-223, 2020.

9. Sánchez-Gómez A, Cevallos W, Grijalva MJ, Silva-Ayçaguer LC, Tamayo S, Jacobson JO, Costales JA, Jiménez-Garcia R, Hernández-Barrera V, Serruya S, et al: Social factors associated with use of prenatal care in Ecuador. Rev Panam Salud Publica 40: 341-346, 2016.

10. Parra-Pingel PE, Quisiguiña-Avellán LA, Hidalgo L, Chedraui $P$ and Pérez-López FR: Pregnancy outcomes in younger and older adolescent mothers with severe preeclampsia. Adolesc Health Med Ther 8: 81-86, 2017.

11. Svanemyr J, Guijarro S, Riveros BB and Chandra-Mouli V: The health status of adolescents in Ecuador and the country's response to the need for differentiated healthcare for adolescents. Reprod Health 14: 29, 2017.

12. Paladugu RK, Donipudi PC, Chimata D and Jasti M: Adolescent pregnancy and its outcomes: A cross-sectional study. Int J Comm Med Pub Health 5: 4408-4414, 2018.

13. Rogers MM, Peoples-Sheps MD and Suchindran C: Impact of a social support program on teenage prenatal care use and pregnancy outcomes. J Adolesc Health 19: 132-140, 1996.

14. Bohîlțea RE, Zugravu CA, Neacsu A, Navolan D, Berceanu C, Nemescu D, Bodean O, Turcan N, Baros Al and Cîrstoiu MM: The prevalence of vitamin D deficiency and its obstetrical effects. A prospective study on Romanian patients. Rev Chim 70: 1228-1233, 2019.

15. Tyrberg RB, Blomberg $M$ and Kjølhede P: Deliveries among teenage women - with emphasis on incidence and mode of delivery: A Swedish national survey from 1973 to 2010 . BMC Pregnancy Childbirth 13: 204-214, 2013.

16. Munteanu O, Ispas A, Berceanu C and Vladareanu S: Development of scoring system for evaluating the risk of vephalopelvic disproportion. Res Sci Today 7: 120-125, 2014.

17. Jain P and Gupta M: Maternal outcome in teenage pregnancy Indian J Obstet Gynecol Res 6: 263-267, 2019.

18. Levy DM: Emergency Caesarean section: Best practice. Anaesthesia 61: 786-791, 2006.

19. Smith D, Stringer E, Vladutiu CJ, Zink AH and Strauss R: Risk of uterine rupture among women attempting vaginal birth after cesarean with an unknown uterine scar. Am J Obstet Gynecol 213: 80.e1-80.e5, 2015. 